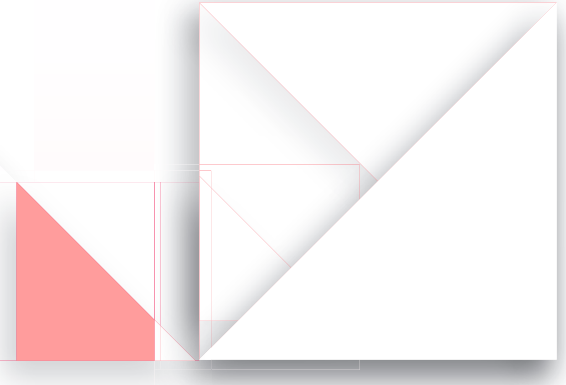

\title{
Estado del arte de la 3 investigación en educación en Colombia: Un caso de los programas de formación doctoral en la nación
}

\section{- The State of the Art of Research in Education in Sciences in Colombia: The Case of Doctorate Training Programs in the Country}

- Estado da arte na pesquisa em educação em ciências na colômbia: o caso dos programas de formação doutoral na nação

\section{Resumen}

Este artículo presenta un estudio acerca del estatuto epistemológico de la investigación en educación en ciencias ofrecida en el país, a través del análisis de los programas de investigación comprendidos en el periodo 2000-2011. Se consideran las hipótesis de investigación desarrolladas por los profesores-investigadores como insumos de análisis, realizando un estudio sobre las tendencias en investigación a nivel internacional en educación en ciencias, para contrastarlas con las investigaciones realizadas en los programas doctorales nacionales. Se recopilan datos según las estructuras curriculares de los programas doctorales, desarrollando entrevistas en profundidad a doctores del área en Educación en Ciencias, implementando y diseñando cuestionarios sobre el campo específico, para finalmente compararlo con líneas de investigación desarrolladas en el tema. Los resultados, muestran que los programas doctorales de la nación abordan el campo de la educación en ciencias en forma limitada e incompleta, siendo el Doctorado Interinstitucional en Educación, por su naturaleza, el más favorecido.

Palabras clave

Campo de la educación en ciencias; líneas de investigación; programas de investigación doctoral

\section{Abstract}

This article presents a study about the epistemological status of research in science education offered in the country, by the analysis of the research programs included in the period 2000-2011. It considers research hypotheses

\section{Alfonso Claret Zambrano** Tatiana Iveth Salazar*** Boris Fernando Candela ${ }^{* * *}$ Leidy Yurani Villa ${ }^{* \star \star \star *}$}

Doctorado, Universidad del Valle; Coordinador Doctorado Interinstitucional en Educación; Posdoctorado in Research in Epistemology, History, Curriculum and Overview; University College London, Institute of Education. Cali, Colombia. Correo electrónico: Alfonso.zambrano@correounivalle.edu.co, Orcid: 0000-0003-4785-6037

Magíster en Educación con énfasis en Enseñanza de las Ciencias, Universidad Estatal Paulista (Unesp), estudiante del Doctorado en Educación para la Ciencia, Bauru-Brasil. Correo electrónico: tativsalo@hotmail.com. Orcid: 0000-0003-3890-8869.

Magíster en Educación con énfasis en Enseñanza de las Ciencias, Universidad del Valle, profesor de la misma universidad. Cali, Colombia. Correo electrónico: bofeca65@yahoo.com, Orcid 0000-0002-5833-1975.

Magíster en Educación, profesora de la Universidad del Valle, Cali, Colombia. Correo electrónico: Leidyvilla010@hotmail.com.Orcid: 0000-0002-7080-929x

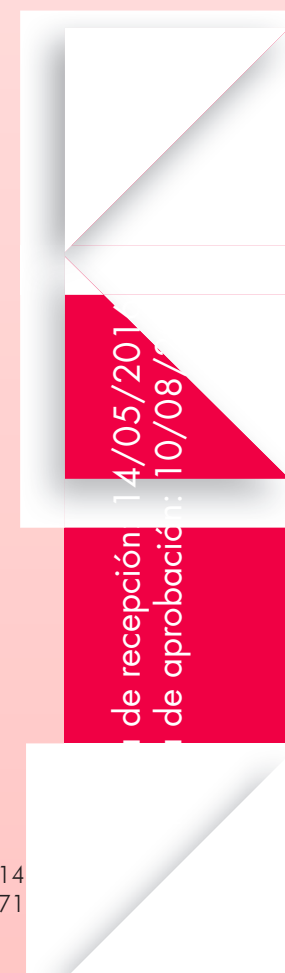


developed by the teachers-researchers as analytical inputs, conducting a study of the international trends in research in science education, in order to compare them with the research carried out in national doctorate programs. Data is collected according to the curricular structures of the doctoral programs by carrying out in-depth interviews with doctors in the field of Science Education, and by implementing and designing questionnaires specific to the field, and then compare them with lines of research developed in the subject. Results show that doctoral programs in the country address the field of science education in a limited and incomplete way, where the Interinstitutional Doctorate in Education is the most favored one due to its nature.

Keywords

Field of science education; lines of research; doctorate research programs

\section{Resumo}

Este artigo apresenta um estudo sobre o estatuto epistemológico da pesquisa em Educação em Ciências oferecida na Colômbia através da análise dos programas de pesquisa compreendidos no período de 2000 a 2011 . Consideram-se as hipóteses de pesquisas desenvolvidas pelos professores-pesquisadores como insumos de análise, realizando um estudo sobre as tendências na pesquisa a nível internacional em Educação em Ciências, para contrastá-las com as pesquisas realizadas nos programas doutorais nacionais. Os dados foram recopilados segundo as estruturas curriculares dos programas doutorais, desenvolvendo entrevistas em profundidade com doutores da área de Educação em Ciências, implementando e fazendo questionários sobre o campo específico, para finalmente compará-los com linhas de pesquisa desenvolvidas no tema. Os resultados evidenciam que os programas doutorais nacionais abrangem o campo da Educação em Ciências de forma limitada e incompleta, sendo o Doutorado Interinstitucional em Educação o mais favorecido.

Palavras chave

Campo da educação em ciências; linhas de pesquisa; programas de pesquisa doutoral 


\section{Introducción}

Este artículo emerge como continuación de una pregunta de investigación previamente planteada en un artículo anterior (Zambrano, Salazar, Candela y Villa, 2013): ¿Cuál es el estatuto epistemológico de la investigación en Educación en ciencias que ofrecemos en la nación, a través de nuestros programas de investigación, en el periodo 2000-2011? En este estudio nos enfocamos hacia los programas doctorales en educación en ciencias, por su impacto conceptual en la formación de educadores en la enseñanza de las ciencias en el país.

En el contexto anterior, la pregunta de investigación es entonces: ¿̇Cuál es el estado de la investigación en la educación en ciencias que ofrecemos en la nación, a través de nuestros programas de investigación doctoral durante el periodo 2000-201 1 ? Este pregunta institucionalmente comprende el análisis de las líneas de investigación que orientan la formación educativa en las ciencias desde pregrado hasta posgrado en programas de doctorado en educación en ciencias naturales, trabajos de grado en las licenciaturas en educación en ciencias y educación ambiental, trabajos de especializaciones, tesis de maestría y doctorado en educación en ciencias. Tratar de resolver la pregunta anterior implica definir teóricamente lo que significa el campo de la educación en ciencias y asumir como práctica la hipótesis de investigación.

El campo se considera el colectivo de las líneas de investigación trazadas por la comunidad de investigadores de educación en ciencias que históricamente las han construido según sus intereses explicativos, sus resultados conceptuales, la sabiduría acumulada y la actividad profesional desarrollada (Toulmin, 1972).

Según lo anterior se hace necesario saber cuáles son las líneas de investigación propias del campo de la educación en ciencias que se han construido en la nación durante el periodo en cuestión. De allí la importancia de elaborar el estado del arte de la actividad investigativa en educación en ciencias realizada en Colombia en la primera década del siglo xxl, a partir del levantamiento del mismo efectuado por iniciativa de Colciencias en asocio con Socolpe en el año 2000. Durante el periodo 2000-201 1 se han producido en Colombia desarrollos importantes en los programas de formación de investigadores en esta área, entre los que destaca la emergencia de programas de doctorado en educación, específicamente orientados hacia la educación en ciencias naturales.

Igualmente, las dinámicas en los contextos educativos nacional e internacional han llevado a desarrollos normativos como los estándares básicos en competencias, que han debido reflejarse en la investigación educativa realizada en Colombia. Por ello, es conveniente efectuar un balance analítico de esta actividad, en sí misma y en su relación con las prácticas educativas. Para este efecto se propone estudiar sistemáticamente la producción científica en esta área del conocimiento: la educación en ciencias y la didáctica de las ciencias. En el caso de esta investigación nos ocuparemos de la educación en ciencias realizada en Colombia en la década pasada, en especial en las universidades en las que se ofrecen los programas de licenciatura, maestría y doctorado en educación en ciencias. De esta manera se espera evidenciar el impacto que ha tenido la investigación en la formación de docentes por medio de las líneas de investigación del campo mencionado.

El saber acerca de las líneas de investigación en la formación de educadores desde el pregrado hasta el doctorado contribuirá a la reflexión, el análisis y el replanteamiento de problemas concretos de enseñanza y 
aprendizaje de las ciencias para luego tratarlos como objeto de investigación en una formación académica superior o de posgrado.

En este sentido, el principal objetivo es analizar los programas de investigación relacionados con la formación de educadores que actualmente se desarrollan en el país pero que mantienen una línea de continuidad institucional desde el pregrado hasta el doctorado.

\section{Antecedentes}

"Líneas de investigación de educación en ciencias en Colombia" (Zambrano, Salazar, Candela y Villa, 2013) es el estudio más valioso, específicamente por sus resultados que son la base del proyecto actual. Ellos constituyen la primera aproximación a las líneas de investigación en el campo de la educación en ciencias en Colombia. Este conjunto de líneas de investigación constituyen el estatuto epistemológico del campo de la educación en ciencias y han sido el producto de varios estudios desarrollados y publicados previamente, algunos de los cuales se enuncian a continuación.

El estudio de Hernández (2000) titulado Aproximación a un estado del arte de la educación en ciencias en Colombia es el resultado de una revisión de las investigaciones apoyadas por Colciencias entre 1988 y 1999. La obra colectiva Educación y formación del pensamiento científico: Cátedra Icfes Agustín Nieto Caballero, editada por A. C. Zambrano (2003), representa el estado de la didáctica en ciencias naturales' en la educación superior. El texto fue escrito por un grupo de profesores universitarios; en él se reseñaron las líneas de investigación hacia las cuales se orientaban las investigaciones en este campo en el contexto nacional. Por último, mencionamos el estudio "Enseñanza de la Biología żun campo de conocimiento? Estado del arte 2007-2008", realizado por Valbuena, Amórtegui y Correa (2012).

\section{Marco teórico o conceptual}

Los resultados del proyecto de líneas de investigación muestran que las que estructuran el campo de la educación en ciencias en el periodo 2000-2011 en la nación son las siguientes:

- La relación entre el conocimiento científico y el conocimiento común.

- Enseñanza, aprendizaje y evaluación: metacognición, cambio conceptual, resolución de problemas e historia de las ciencias.

- La relación entre la teoría y la práctica en las ciencias experimentales a través del laboratorio experimental.

1 En este texto, escrito en 2003, se asumía la didáctica de las ciencias como el único campo que justificaba la enseñanza, el aprendizaje y la evaluación de las ciencias naturales, producto de la enculturación española. 
- Las nuevas tecnologías de la informática y la comunicación, y su relación con la educación en ciencias naturales. Inteligencia artificial y procesos de razonamiento.

- Desarrollo curricular de las ciencias naturales.

- Contextos culturales, educación ambiental, educación en ciencias en ambientes no convencionales.

- Conocimiento, pensamiento y formación del maestro.

Estos resultados muestran una gran diferencia entre lo que se hace a nivel nacional e internacional en investigación en educación en ciencias. Se dejan de lado algunas líneas que están en permanente transformación, como Ciencia, Tecnología y Sociedad (СтS), que ahora evoluciona hacia Ciencia, Tecnología, Sociedad y Ambiente (CTS-A), o los valores de la ciencia, o la naturaleza de la ciencia, cosmovisión o ciencia, tecnología, ingeniería y matemática (STEM, por sus siglas en inglés). También dejan en evidencia un gran problema conceptual aún sin resolver: ¿̇Cuál es el significado de línea de investigación? De allí nuestra propuesta de conceptualizar acerca de la naturaleza de una línea de investigación, específicamente por la importancia que tiene para el proyecto. Por lo anterior, la línea de investigación (LDI) se comprende como el conjunto de problemas, referentes teóricos, métodos de indagación, hipótesis que se proponen y las soluciones que se desarrollan, en el campo de la educación en ciencias, cuyo proceso global contribuye a incrementar el conocimiento en dicho campo disciplinar.

La línea de investigación es un proceso que consiste en resolver un problema o conjunto de problemas educativos en las ciencias por medio de un método determinado en el tiempo, cuyos resultados contribuyen a la elaboración conceptual del campo de la educación en ciencias en la sociedad, la región, la institución y en el aula. Como proceso en permanente construcción tiene un inicio conceptual (su documento de fundación como referencia para su evolución) y uno final de desarrollo para su contrastación conceptual. En ese contraste, la LDI produce conceptos, teorías, métodos, otros problemas, publicaciones, textos, artículos, ponencias, hace escuela, esto es, hace discípulos, forma escolares en esa línea en el mejor de los sentidos. Una LDI evoluciona, pero a diferencia del sujeto orgánico que la funda, que la establece, es inmortal; no muere en este espacio y tiempo newtoniano, pertenece al tiempo y al espacio einsteiniano. La LDI supera su sujeto fundador y por último se organiza en torno a trabajos de grado, trabajos de investigación en maestrías y disertaciones de doctorado. En síntesis, la LDI con sus preguntas de investigación constituye el estatuto epistemológico del campo de la educación en ciencias porque ellas justifican su contenido.

\section{Metodología}

Ofrecer una respuesta al problema objeto de este estudio implica conocer lo que se está realizando a nivel internacional en la investigación en la educación en ciencias, durante el mismo periodo, y contrastarlo con las investigaciones que se desarrollan a nivel nacional que se están considerando para el caso particular, teniendo como referencia las investigaciones acerca del tema que se realizaron en ese periodo con el apoyo de Colciencias, las que se desarrollaron en las instituciones educativas y las que se presentaron en eventos nacionales e internacionales, de acuerdo con las líneas de investigación planteadas por los profesores, directores y participantes de esos proyectos. Es decir, conociendo cuáles son las hipótesis de investigación que plantean 
los profesores investigadores, desde su formación inicial hasta la actualidad de su formación educativa, sabremos acerca de las propuestas de investigación generadas con relación a las tesis de doctorado, maestrías y pregrado sobre educación en ciencias en las universidades que guardan una línea de investigación continua en la formación en dicho campo desde el pregrado hasta el doctorado. Se definen cuatro preguntas de trabajo: qué se investiga, cómo se investiga, qué resultados se obtienen y qué significa lo anterior para el estatuto epistemológico de la investigación y para las prácticas pedagógicas.

La pregunta de investigación que se plantea el proyecto exige integrar tanto el paradigma cuantitativo como el cualitativo, pero sobre todo triangular teorías, datos y métodos de investigación en el estudio de la investigación generada desde los diversos programas considerados en Colciencias, programas de doctorado y programas de formación en investigación en pregrado y posgrado (Blanchet, Ghiglione, Massonat, y Trognon, 1989; Deslauriers, 2004; Kerlinger, 2002; Latorre, del Rincón, y Arnal, 1996; Maxwell, 2005; Rodríguez, Gil y García, 1996). En particular, se tomarán como modelos para el diseño metodológico los trabajos cuyos objetivos son similares, bien sea el establecimiento del estado del arte de la investigación educativa (por ejemplo, Hernández 2000, y los demás trabajos que lo acompañan para otras áreas curriculares), o bien el estado de los estudios doctorales en educación en ciencias en un sistema educativo (Munari, 1999), como el estudio de Jablon (2002), referido al contexto norteamericano.

Así pues, las técnicas de investigación que se utilizarán para recoger los datos serán el estudio documental, las entrevistas en profundidad, los cuestionarios y la rejilla de análisis de contenido (Abela, 2000). De acuerdo a lo anterior planteamos como dispositivo metodológico un análisis global del DIE, sus estructuras curriculares, las líneas de investigación ofrecidas con relación al campo de la educación en ciencias y la documentación pertinente que describa los programas de doctorado en educación en ciencias en las instituciones que tienen esta secuencia completa desde el pregrado hasta el posgrado.

\section{Análisis y resultados}

Para establecer el estado del arte de la investigación en educación en ciencias en Colombia, es necesario identificar los programas de pregrado, maestría y doctorado que se ofrecen en las universidades oficiales y privadas con registro calificado o acreditación de alta calidad, con el objetivo de ubicar un panorama general de la formación de profesionales e investigadores de la educación en ciencias naturales del país. Asimismo, es preciso analizar las universidades que ofrecen la línea de formación completa de licenciatura, maestría y doctorado, estudiando sus objetivos, las estructuras curriculares y líneas de investigación que sustentan su acción de formación. 
De acuerdo a lo anterior, los programas de doctorado que funcionan en Colombia se clasifican de la siguiente manera: (1) Doctorado Interinstitucional en Educación (DIE), que articula a la Universidad Distrital Francisco José de Caldas, la Universidad Pedagógica Nacional y la Universidad del Valle. (2) Doctorado en Ciencias de la Educación -Red de Universidades Estatales de Colombia, RudeColombia-, que reúne a las universidades del Tolima, Quindío, Magdalena, Cauca, Atlántico, Nariño, Cartagena, la Tecnológica de Pereira y la Tecnológica y Pedagógica de Colombia. (3) Las universidades de Antioquia, los Andes (privada), La Salle (privada), Santo Tomas (privada), Caldas, Simón Bolívar y Fundación Universitaria del Norte (privada) funcionan de manera independiente. En el anexo 2 se muestran las universidades con la línea completa, según su formación se extienda desde pregrado hasta posgrado. Para efectos del análisis de los programas de doctorado se tendrán en cuenta aquellos que se ofrecen en las universidades con la línea de formación completa, estos son: El DIE, RudeColombia y el Doctorado en Educación de la Universidad de Antioquia.

Estos programas de Doctorado en Educación con énfasis en Educación en Ciencias del país permiten pensar este campo disciplinar como referencia conceptual para formular los objetivos, desarrollar las líneas de investigación y diseñar las estructuras curriculares. Dicho campo se estructura con las bases epistemológicas mencionadas en el marco teórico, de ahí que sea necesario establecer las tendencias de estos programas con respecto a cada una de las unidades de registro, a saber: visión, misión, propósitos, perfil del estudiante, perfil del egresado y líneas de investigación.

Con el propósito de analizar las estructuras curriculares que plantean para el desarrollo de los programas de doctorado en educación, a continuación se presenta, en primer lugar la estructura conceptual del campo disciplinar de la educación en ciencias y luego la organización curricular de los doctorados de la Universidad de Antioquia, el DIE y el de Rudecolombia con base en dicha estructura. Estos tres programas son los que se analizarán en esta sección del artículo debido a que cumplen con la línea completa de formación de pregrado, maestría y doctorado

\section{Las estructuras curriculares}

Las estructuras curriculares se organizan jerárquicamente según las categorías que se asumen del conocimiento de la educación en ciencias, así: misión, visión, propósitos, perfil del estudiante, perfil del egresado y líneas de investigación. Con respecto a esta forma organizativa son idénticas, pero su contenido difiere. Veamos de cerca estas relaciones y diferencias entre las universidades seleccionadas.

\section{Misión y visión}

El DIE propone afianzar y desarrollar el campo intelectual de la educación. RudeColombia propone teorizar e intervenir los factores de las culturas socioeducativas y sugiere ser un interlocutor válido en la política pública de educación. La Universidad de Antioquia propone producción de conocimiento de la educación que el país requiere, asumiendo la educación como una prioridad científica, social y cultural.

El primer proyecto asume una misión del campo intelectual de la educación, el segundo plantea una misión de educación social-cultural, y el tercero, una de educación científica.

\section{Propósitos y objetivos}

Las tres estructuras coinciden en desarrollar la investigación, pero proponen hacerlo de 
distinta forma. El DIE contribuye al avance del conocimiento científico en el campo de la educación, RudeColombia orienta la investigación hacia la problemática educativa colombiana y latinoamericana desde la internacionalización del conocimiento, y la Universidad de Antioquia aporta al avance del conocimiento científico en el campo de la educación. No obstante, en estos propósitos de investigación no se encuentra de manera explícita un elemento que desempeña un papel clave en el desarrollo profesional de los doctorandos: la formación en el conocimiento necesario para el proceso de enseñanza-aprendizaje y la evaluación en el aula. En síntesis, las estructuras curriculares están más orientadas a la formación de investigadores del campo de la educación en ciencias, que si bien es un componente fundamental de la formación, deja de lado la importancia de pensar la formación como profesores de educación en ciencias (Abell, 2009 y Jablon, 2002).

\section{Perfil del estudiante}

Los tres programas exigen requisitos regulares de admisión, tales como estudios previos de maestría, dedicación al doctorado y ubicarse en una línea de investigación.

\section{Perfil del egresado}

El DIE propone investigar en educación, pedagogía y didáctica, y relaciones con el área del conocimiento. RudeColombia propone investigar en el área de un campo de conocimiento. Antioquia, por último, plantea formar científicos en el campo de la educación en ciencias.

\section{Líneas de investigación}

Se muestran diferencias profundas entre los tres programas de doctorado. El DIE aborda diecisiete, RudeColombia, dos y la Universidad de Antioquia, cuatro.

Como puede evidenciarse en estas secciones de las estructuras curriculares presentadas, se identifica que estas se organizan a través de componentes, los cuales al ser interpretados pueden agruparse bajo unas categorías que permiten establecer que los doctorados en educación tienen en cuenta componentes similares (véase la tabla 1).

Tabla 1. Componentes de las estructuras curriculares

\begin{tabular}{|l|l|l|l|}
\hline \multicolumn{4}{|c|}{ Componentes de las estructuras curriculares } \\
\hline \multicolumn{1}{|c|}{ Categoría } & \multicolumn{1}{|c|}{ DIE } & \multicolumn{1}{c|}{ RudeColombia } & \multicolumn{1}{c|}{ Antioquia } \\
\hline $\begin{array}{l}\text { Componente } \\
\text { pedagógico }\end{array}$ & Educación y pedagogía & Saber pedagógico & Teoría \\
\hline $\begin{array}{l}\text { Componente } \\
\text { investigativo }\end{array}$ & Investigación & $\begin{array}{l}\text { Investigación y } \\
\text { producción }\end{array}$ & Investigación \\
\hline $\begin{array}{l}\text { Componente } \\
\text { complementario/ } \\
\text { disciplinar }\end{array}$ & Énfasis & $\begin{array}{l}\text { Profundización } \\
\text { disciplinaria }\end{array}$ & Complementaria \\
\hline
\end{tabular}

Fuente: elaboración propia 
En la organización de los doctorados en educación se identifica que todos ellos se estructuran desde tres componentes fundamentales: el pedagógico, el investigativo y el complementario/ campo en el cual se aglutina la formación disciplinar y la formación complementaria. En este sentido se da gran relevancia a la investigación en el programa; el proyecto de investigación de los doctorandos presenta el mayor porcentaje y énfasis.

\section{Análisis de las líneas de investigación de los doctorados con relación a las bases epistemológicas}

El análisis de las líneas de investigación que orientan los programas de formación doctoral se presenta en función de las bases epistemológicas que constituyen el campo de la educación en ciencias naturales. El propósito de incluirlas aquí es mostrar la diversidad de las líneas de investigación como respuesta al problema central del campo de la educación en ciencias (Gabel, 1994; Linn, 1987; Shulman, 1987; Zambrano, 2014) (véase la tabla 2). Esta diversidad debe explicitarse en la sección donde se presenta la estructura del campo de la educación en ciencias.

Tabla 2. Análisis de las líneas de investigación de los programas de doctorado en Colombia

\begin{tabular}{|c|c|c|c|c|}
\hline \multicolumn{5}{|c|}{ AGRUPACIÓN LÍNEAS DE INVESTIGACIÓN } \\
\hline $\begin{array}{c}\text { Base } \\
\text { epistemológica }\end{array}$ & DIE & Antioquia & RudeColombia & Análisis \\
\hline $\begin{array}{l}\text { ¿Qué } \\
\text { conocimiento es } \\
\text { necesario para la } \\
\text { enseñanza en el } \\
\text { campo disciplinar } \\
\text { de la educación en } \\
\text { ciencia? }\end{array}$ & $\begin{array}{l}\text { - Epistemología, Historia y } \\
\text { Desarrollo Curricular en las } \\
\text { Ciencias (Uv) } \\
\text { - Relación entre Historia y } \\
\text { Filosofía de las Ciencias y } \\
\text { la Didáctica de las Ciencias } \\
\text { (UD) } \\
\text { - Historia, Filosofía y } \\
\text { Enseñanza de las Ciencias } \\
\text { (UV) } \\
\text { - La Formación Científica en } \\
\text { los Lugares Públicos (UPN) }\end{array}$ & $\begin{array}{l}\text { - Historia y } \\
\text { Epistemología } \\
\text { y Educación } \\
\text { de las Ciencias } \\
\text { Experimentales } \\
\text { - Relación } \\
\text { Escuela-Museo }\end{array}$ & & $\begin{array}{l}\text { El doctorado de } \\
\text { RudeColombia no presenta } \\
\text { una línea de investigación } \\
\text { que se fundamente en } \\
\text { la base epistemológica } \\
\text { relacionada con la enseñanza } \\
\text { en el campo de la educación } \\
\text { en ciencias }\end{array}$ \\
\hline $\begin{array}{l}\text { ¿Cómo se } \\
\text { aprenden las } \\
\text { ciencias? }\end{array}$ & $\begin{array}{l}\text { - } \text { Formación en } \\
\text { Competencias Científicas } \\
\text { (Uv) } \\
\text { - Epistemología, Historia y } \\
\text { Desarrollo Curricular en las } \\
\text { Ciencias (UV) } \\
\text { - Relación entre historia y } \\
\text { Filosofía de las Ciencias y } \\
\text { la Didáctica de las Ciencias } \\
\text { (UD) } \\
\text { - Historia, Filosofía y } \\
\text { Enseñanza de las Ciencias } \\
\text { (Uv) } \\
\text { - Estilo Cognitivo y Logro } \\
\text { Educativo (UPN) } \\
\text { - Ritmos Cognitivos (UPN) } \\
\text { - Estilos Pedagógicos (UPN) } \\
\text { - Elaboración de Conceptos } \\
\text { Científicos (UPN) } \\
\text { - La Formación Científica en } \\
\text { los Lugares Públicos (UPN) }\end{array}$ & $\begin{array}{l}\text { - Aprendizaje } \\
\text { por Cambio } \\
\text { Conceptual y } \\
\text { Educación En } \\
\text { Ciencias } \\
\text { - Relación } \\
\text { Escuela-Museo }\end{array}$ & & $\begin{array}{l}\text { La base epistemológica que } \\
\text { se refiere al aprendizaje en } \\
\text { la educación en ciencias } \\
\text { se está desarrollando en } \\
\text { el DIE a través de ocho } \\
\text { líneas de investigación; } \\
\text { en el doctorado de la } \\
\text { Universidad de Antioquia } \\
\text { se desarrolla una única } \\
\text { línea de investigación y en } \\
\text { RudeColombia no se está } \\
\text { desarrollando esta base } \\
\text { epistemológica. }\end{array}$ \\
\hline
\end{tabular}




\begin{tabular}{|c|c|c|c|c|}
\hline \multicolumn{5}{|c|}{ AGRUPACIÓN LÍNEAS DE INVESTIGACIÓN } \\
\hline $\begin{array}{c}\text { Base } \\
\text { epistemológica }\end{array}$ & DIE & Antioquia & RudeColombia & Análisis \\
\hline $\begin{array}{l}\text { ¿Cómo se } \\
\text { relaciona la } \\
\text { ciencia, la } \\
\text { tecnología la } \\
\text { sociedad en la } \\
\text { educación en } \\
\text { ciencias? }\end{array}$ & $\begin{array}{l}\text { - Educación Ambiental y } \\
\text { Desarrollo Sostenible (UV) } \\
\text { - Ciencia, Tecnología y } \\
\text { Sociedad (UPN) } \\
\text { - } \text { Ciencia, Tecnología y } \\
\text { Sociedad (UD) } \\
\text { - Historia social de las } \\
\text { Ciencias y las Tecnologías } \\
\text { (UD) }\end{array}$ & & & $\begin{array}{l}\text { De acuerdo con los datos } \\
\text { presentados en esta tabla } \\
\text { se identifica que solo el } \\
\text { doctorado del DIE está } \\
\text { desarrollando esta base } \\
\text { epistemológica }\end{array}$ \\
\hline $\begin{array}{l}\text { ¿Qué, cómo, para } \\
\text { qué, con qué y a } \\
\text { quién se evalúa } \\
\text { en el campo de } \\
\text { la educación en } \\
\text { ciencias? }\end{array}$ & $\begin{array}{l}\text { - Evaluación en Ciencias (UPN) } \\
\text { - Formación en } \\
\text { Competencias Científicas } \\
\text { (Uv) }\end{array}$ & & & $\begin{array}{l}\text { La evaluación, a pesar de } \\
\text { seruna base epistemológica } \\
\text { fundamental del campo de } \\
\text { la educación en ciencias, } \\
\text { únicamente la aborda el } \\
\text { programa del DIE. }\end{array}$ \\
\hline $\begin{array}{l}\text { ¿Cómo debe ser } \\
\text { la formación de } \\
\text { educadores en el } \\
\text { campo disciplinar } \\
\text { de la educación } \\
\text { en ciencias? }\end{array}$ & $\begin{array}{l}\text { - Conocimiento Profesional } \\
\text { del Profesor de Ciencias } \\
\text { (UPN) } \\
\text { - Conocimiento Profesional } \\
\text { de los Profesores de } \\
\text { Ciencias y Conocimiento } \\
\text { Escolar (UPN) } \\
\text { - Conocimiento Profesional } \\
\text { de los Profesores de } \\
\text { Ciencias y el Conocimiento } \\
\text { Escolar (UD) } \\
\text { - Cambio Didáctico y } \\
\text { Formación del Profesorado } \\
\text { de Ciencias (UD) } \\
\text { Ciencia, Acciones y } \\
\text { Creencias (UPN) }\end{array}$ & $\begin{array}{l}\text { Formación } \\
\text { de Profesores } \\
\text { de Ciencias } \\
\text { Experimentales }\end{array}$ & $\begin{array}{l}\text { Didáctica de las } \\
\text { Ciencias }\end{array}$ & $\begin{array}{l}\text { Se considera que la } \\
\text { formación de los profesores } \\
\text { está afectando directamente } \\
\text { el aula; en este sentido, } \\
\text { en los tres programas de } \\
\text { doctorado analizados existen } \\
\text { líneas de investigación que } \\
\text { a través de su desarrollo } \\
\text { permiten fortalecer la } \\
\text { base epistemológica de la } \\
\text { formación de educadores. }\end{array}$ \\
\hline $\begin{array}{l}\text { ¿Cómo influye } \\
\text { el contexto } \\
\text { sociocultural } \\
\text { en el proceso } \\
\text { enseñanza- } \\
\text { aprendizaje en } \\
\text { la educación en } \\
\text { ciencias? }\end{array}$ & $\begin{array}{l}\text { - Enseñanza de las Ciencias y } \\
\text { Contexto Cultural (UPN) } \\
\text { - Enseñanza de las Ciencias, } \\
\text { Contexto y Diversidad } \\
\text { Cultural (UD) }\end{array}$ & & & $\begin{array}{l}\text { El desarrollo de esta base } \\
\text { epistemológica está iniciando } \\
\text { su proceso, por ende } \\
\text { podemos observar que el DIE } \\
\text { es el único que desarrolla } \\
\text { dos líneas de investigación en } \\
\text { función de esta base. }\end{array}$ \\
\hline $\begin{array}{l}\text { ¿Cómo se } \\
\text { seleccionan, } \\
\text { secuencian y } \\
\text { temporalizan } \\
\text { los contenidos y } \\
\text { principios de las } \\
\text { ciencias naturales? }\end{array}$ & $\begin{array}{l}\text { Epistemología, Historia y } \\
\text { Desarrollo Curricular en las } \\
\text { Ciencias (UV) }\end{array}$ & & $\begin{array}{l}\text { Currículo y } \\
\text { Sociedad } \\
\text { Sujeto, Saber } \\
\text { Pedagógico y } \\
\text { Ciencia }\end{array}$ & $\begin{array}{l}\text { El currículo que marca una } \\
\text { distinción entre educación en } \\
\text { ciencias y la didáctica de las } \\
\text { ciencias no se abordao en el } \\
\text { doctorado de la Universidad } \\
\text { de Antioquia. }\end{array}$ \\
\hline
\end{tabular}

Fuente: elaboración Propia 


\section{Bases epistemológicas que no son desarrolladas por ninguno de los doctorados en educación estudiados}

\section{Tabla 3. Bases epistemológicas no desarrolladas por los doctorados}

\begin{tabular}{|c|c|}
\hline Base epistemológicas que no son utilizadas & Análisis \\
\hline $\begin{array}{l}\text { ¿Cómo influyen las políticas educativas estatales en el campo } \\
\text { disciplinar de la educación en ciencias? }\end{array}$ & $\begin{array}{l}\text { Uno de los propósitos fundamentales de la formación doctoral } \\
\text { es incidir en las políticas del Estado, sin embargo se identifica } \\
\text { que estas no están siendo abordadas de manera explícita por } \\
\text { ninguno de los programas de doctorado analizados. } \\
\text { En la visión del programa de doctorado de RudeColombia se } \\
\text { plantea "Ser interlocutor válido en las políticas públicas de } \\
\text { la educación"; no obstante, carece de líneas de investigación } \\
\text { enfocadas al estudio de la política educativa. } \\
\text { Una posible conclusión es que la universidad y el Estado } \\
\text { no están participando conjuntamente en la construcción de } \\
\text { políticas educativas para la sociedad colombiana. Por ende, } \\
\text { se carece de debate político en los programas doctorales. }\end{array}$ \\
\hline $\begin{array}{l}\text { ¿Qué teorías educativas orientan la educación en ciencias? } \\
\text { (TP- TI-TC) }\end{array}$ & $\begin{array}{l}\text { A pesar de que en la misión del programa de doctorado } \\
\text { de la Universidad de Antioquia se explicita: "Consolidar } \\
\text { teorías pedagógicas y didácticas que constituyan la base } \\
\text { de las soluciones a las problemáticas educativas...", en } \\
\text { la del DIE se plantea "[...] la construcción colectiva de } \\
\text { un pensamiento pedagógico", y RudeColombia plantea } \\
\text { "formar investigadores con autonomía intelectual para } \\
\text { comprender, teorizar e intervenir los factores de las culturas } \\
\text { socioeducativas", la intención de aportar a la construcción de } \\
\text { la teoría educativa en el país no se materializa directamente } \\
\text { en el desarrollo de las diferentes líneas de investigación en } \\
\text { educación en ciencias. }\end{array}$ \\
\hline $\begin{array}{l}\text { ¿Cuáles son las finalidades que se plantean en el campo } \\
\text { disciplinar de la educación en ciencias? }\end{array}$ & $\begin{array}{l}\text { Desde el punto de vista de los propósitos de los programas } \\
\text { de doctorado se nota la relación existente con la base } \\
\text { epistemológica en consideración; sin embargo, el análisis de } \\
\text { las líneas de investigación demuestra que las finalidades de la } \\
\text { educación en ciencias no son objeto de investigación directo, } \\
\text { solo indirecto. }\end{array}$ \\
\hline ¿Cómo se investiga en la educación en ciencias? & $\begin{array}{l}\text { Los programas doctorales tienen como propósito clave hacer, } \\
\text { desarrollar y aplicar la investigación educativa y efectivamente } \\
\text { lo hacen, pero no reflexionan sobre dicho proceso. }\end{array}$ \\
\hline
\end{tabular}

Fuente: elaboración propia

\section{Conclusiones generales del análisis de los componentes de las estructuras curriculares}

El análisis de los componentes de las estructuras curriculares de los doctorados (DIE, Universidad de Antioquia y RudeColombia) permite evidenciar que estas se fundamentan y se desarrollan en función del campo de la educación en ciencias, y que este se especifica a través de las bases epistemológicas ${ }^{2}$. De ahí que la organización de toda estructura curricular en función de sus cursos, seminarios, investigaciones y prácticas depende de cómo asume la universidad el campo de la educación en ciencias. En este caso, al no tener una concepción homogénea

2 Las bases epistemológicas resultan del cruce de los libros de investigación en Colombia que muestran una visión general del campo de la educación en ciencias; además de esto se logran visualizar a través de las revistas, los proyectos de investigación, las estructuras curriculares y las líneas de investigación que existen en este momento. 
de este, dichas estructuras curriculares no cubren el campo en su totalidad, por el contrario se enfocan hacia las líneas de investigación concretas que tienen los diferentes grupos participantes en el doctorado, lo que permite el avance de estas propuestas de investigación. Ahora bien, las investigaciones obtenidas a través de las tesis de doctorado no necesariamente se enfocan en el desarrollo del campo en su totalidad, sino que desarrollan parcialmente las bases epistemológicas, de acuerdo a sus líneas de investigación.

Desde esta perspectiva, las bases epistemológicas del campo de la educación en ciencias que se trabajan a través de los doctorados son: la enseñanza; el aprendizaje; la evaluación; la relación entre ciencia, tecnología sociedad y ambiente; los recursos utilizados, la formación de educadores, el contexto sociocultural y el currículo de la educación en ciencias (Zambrano, 2010). Por otro lado, los programas de doctorado no abordan todas las bases epistemológicas que conforman el campo de la educación en ciencias; dejan de lado bases como las políticas educativas estatales y las teorías educativas (Moore, 1980), que orientan la Educación en ciencias, las finalidades, la naturaleza de la ciencia, cómo los actores participantes en el acto educativo reflexionan sobre su propio pensamiento y cómo se investiga en la educación en ciencias.

Lo anterior permite suponer que la selección de los elementos teóricos y procedimentales provenientes del campo de la Educación en Ciencias que se recogen en los diferentes programas de doctorado se han llevado a cabo a partir de los siguientes aspectos:

- La formación de los profesores-investigadores. Los profesores-investigadores que dirigen el programa son los que fundamentan y permiten el desarrollo de las líneas de investigación. De esta manera, los sistemas de conocimientos, creencias y valores del profesor-investigador influyen directamente en las estructuras curriculares, dado que es desde su formación que se construye y desarrolla el programa de doctorado.

- Políticas educativas de la institución universitaria. Cabe destacar que las políticas educativas de la(s) institución(es) universitaria(s) que desarrolla(n) el programa influyen en las estructuras curriculares. En este caso, por ejemplo, la articulación con otras universidades nacionales e internacionales facilita el intercambio de estudiantes y profesores para el desarrollo del programa.

- Intereses de los doctorandos. Debido a que el programa de formación doctoral debe permitir que los estudiantes desarrollen de manera adecuada su proyecto de investigación, estos intereses normalmente están en función de las líneas de investigación que se promueven en él. Sin embargo, si es pertinente una variación en función de un proyecto se debe plantear. 
- Políticas educativas y de investigación de Estado (Colciencias). Colciencias apoya directamente la investigación en educación en ciencias a través de sus convocatorias (becas de doctorado o convocatorias públicas para proyectos de investigación, donde los doctorandos participan como asistentes de investigación). Las líneas de investigación que este programa ofrece son: (1) Diversidad Étnica y Cultura, Derechos y Políticas Públicas; (2) Política, Estado y Relaciones de Poder; (3) Conflicto, Criminalidad, Derechos, Justicia y Equidad; (4) Desarrollo Humano, Ética y Calidad de Vida; (5) Economía, Innovación, Competitividad y Sostenibilidad; (6) Procesos Espaciales, Dinámicas Sociales y Poblacionales; (7) Comunicación, Información y Cultura; (8) Estudios Sociales sobre Ciencia, Tecnología y Otras Formas de Conocimiento; (9) Estudios de Artes y Humanidades.

En este caso, el hecho de que no se desarrollen algunas bases epistemológicas no se debe a que las omitan conscientemente, sino a que no existe un sujeto, profesor-investigador (en la mayoría de los casos doctor) que posibilite su desarrollo.

Esta situación puede ser explicitada debido a la necesidad de tener los profesores-investigadores suficientes para la apertura del doctorado; en este caso, varios grupos de investigación, doctores y líneas de investigación que a través de sus trabajos permitan el desarrollo del campo de la educación en ciencias, de acuerdo con la manera como los programas de doctorado lo abordan. En este contexto, se supone que el desarrollo de las líneas de investigación en los programas de doctorado en educación en nuestro país permite el desarrollo del campo de la investigación en educación en ciencias. Al respecto, podemos concluir que

- El DIE es el programa que de manera más significativa ayuda al desarrollo del campo, pues sus líneas de investigación fortalecen directamente ocho bases epistemológicas que giran en torno a enseñanza; aprendizaje; evaluación, relación entre ciencia, tecnología, sociedad y ambiente, recursos utilizados, formación; contexto sociocultural y currículo. Esto es posible gracias a que la participación de tres universidades permite la articulación de diversos grupos de investigación y profesores-investigadores que orientan las líneas de investigación. Además, cabe destacar que este programa de doctorado solo se enfoca en la educación en ciencias.

- El doctorado de la Universidad de Antioquia aborda tres bases epistemológicas (enseñanza, aprendizaje y formación) en el desarrollo de sus líneas de investigación.

- El programa de RudeColombia en el área de investigación doctoral que corresponde directamente a la educación en ciencias -en este caso el de Pedagogía, Currículo y Didácticadesarrolla tan solo dos bases epistemológicas (formación y currículo). Es el programa que menos permite el desarrollo del campo al orientar sus investigaciones a dos aspectos concretos de este. No obstante, debemos considerar que el doctorado de RudeColombia plantea otros campos educativos distintos del de la educación en ciencias. El programa se divide en Área de Investigación, Historia de la 
Educación Latinoamericana, Área de Pedagógica, Currículo y Didáctica, Área de Pensamiento Educativo y Comunicación.

Otra conclusión que se deriva de este proyecto es que la investigación que se desarrolla en el país tiene diferentes fuentes: mientras en el primer proyecto donde se establece el estado del arte de la investigación en educación en ciencias, periodo 1988-1999, solo se tomó como referencia los proyectos de Colciencias, este nuevo proyecto analiza tres fuentes diferentes:: (1) a nivel público (revistas nacionales e internacionales, ponencias), institucional (tesis de pregrado, maestría y doctorado), (2) a nivel de convocatoria (estudios financiados por Colciencias), y (3) a nivel institucional. Esto permitió identificar, describir, recoger y contrastar los diferentes resultados obtenidos, para el periodo de análisis (2000-2011).

Este estudio nos permite concluir que las líneas de investigación, el campo de la educación en ciencias y las estructuras curriculares de los programas de doctorado se encuentran articulados para la formación de educadores en ciencias.

Este estudio también nos muestra que los trabajos de pregrado, al igual que los trabajos de investigación de maestría y tesis o disertaciones de doctorado se enmarcan en las líneas de investigación adscritas a los programas de formación, las cuales forman parte de la estructura fundamental del campo disciplinar en educación en ciencias.

El concepto de línea de investigación que se genera desde este estudio permite articular los hallazgos institucionales de investigación, públicos y privados. Finalmente, la investigación muestra que el campo disciplinar se fundamenta en la enseñanza, el aprendizaje y la evaluación de las ciencias naturales como las categorías que atraviesan todas las propuestas de líneas de investigación consideradas en el orden nacional e internacional.

\section{Referencias}

Abela, J. (2000). Las técnicas de análisis de contenido: una revisión actualizada. En: http://public.centrodeestudiosandaluces.es/pdfs/S200103.pdf (fecha de consulta: 2 de septiembre de 2012).

Abell, S. K.; Park Meredith, A. R.; Hanuscin, D. L.; Lee, H. M. y Gagnon, M. J. (2009). Preparing the Next Generation of Science Teacher Educators: A Model for Developing PCK for Teaching Science Teachers. J Sci Teacher Educ, 20, 77-93.

Blanchet, A.; Ghiglione, R.; Massonnat, J. y Trognon, A. (1989). Técnicas de investigación en ciencias sociales. Madrid: Narcea.

Deslauriers, J.-P. (2004). Guía práctica. Jean-Pierre Deslauriers. Profesor de la Universidad de Québec-Hull. Canadá: Pereira, Colombia: Editorial Papiro.

Gabel (1994) (ed.). Handbook of Research on Science Teaching and Learning. Nueva York: MacMillan. 
Hernández (2000). Aproximación a un estado del arte de la educación en ciencias en Colombia. En Estados del arte de la investigación, educación y pedagogía en Colombia. Tomo I. Bogotá: Icfes, Colciencias, Sociedad Colombiana de Pedagogía-Socolpe,

Jablon, P. C. (2002). The status of science education doctoral programs in the United States: The need for core knowledge and skills. Electronic Journal of Science Education, 7(1). Recuperado de http://unr.edu/homepage/ jcannon/ejse/ejse.html.

Kerlinger, F. y Lee, H. (2002). Investigación del comportamiento (4. ${ }^{a}$ ed.). México: McGraw-Hill.

Latorre, A.; Del Rincón, D. y Arnal, J. (1996). Bases metodológicas de la investigación educativa. Barcelona: Gr92.

Linn, M. (1987). Establishing a research base for science education: Challenges, trends and recommendations. Journal of Research in Science Teaching, 24(3), 191-216.

Maxwell, J. A. (2005). Qualitative research design: An interactive approach (2. ${ }^{\mathrm{a} e d}$.). Thousand Oaks, CA: Sage.

Moore, T. (1980). Introducción a la teoría de la educación. Madrid: Alianza.

Munari, A. (1999). Jean Piaget. Unesco: Oficina Internacional de Educación.

Rodríguez, G.; Gil, J. y García, E. (1996). Metodología de la investigación cualitativa. España: Aljibe.

Shulman, L. (1987). Knowledge and teaching: Foundation of the New Reform. Harvard Educational Review, 57(1), 1-21.
Toulmin, S. (1972). Human understanding (vol. 1). "General Introduction" and "Part I". Claredon Press.

Valbuena, E.; Amórtegui, E. y Correa, M. (2012). Enseñanza de la biología: ¿̇un campo de conocimiento? Estado del arte 2007-2008. TEA, 31 (01), 67-90.

Zambrano, A. (2003). Educación y formación del pensamiento científico. Cátedra Icfes Agustín Nieto Caballero. Universidad del Valle.

Zambrano, A. (2010). El currículo como reflexión, investigación y construcción de sujetos en educación. En La formación de educadores en ciencias en el contexło de la investigación en el aula. Santiago de Cali. Educyt.

Zambrano, A. (2014). Estatuto epistemológico de la investigación del campo de la educación en Ciencias en el periodo 2000-201 1. Programa Editorial de la Universidad del Valle.

Zambrano, A.; Salazar, T.; Candela, B. y Villa, L. (2013). Líneas de investigación en educación en ciencias en Colombia. Educyt, 13.

\section{Para citar este artículo}

Zambrano, A.; Salazar, T.; Candela, B. y Villa, L. (2017). Estado del arte de la investigación en educación en Colombia: Un caso de los programas de formación doctoral en la nación. Revista de la Facultad de Ciencia y Tecnología-Tecné, Episteme y Didaxis, TED, 41, 57-71. 